\title{
Analytical solutions of water suction and drying processes vs. experiments
}

\author{
Peter Matiasovsky ${ }^{1, *}$, and Peter Mihalka ${ }^{1}$ \\ ${ }^{1}$ Institute of Construction and Architecture, Slovak Academy of Sciences, Dubravska cesta 9, 84503 Bratislava, Slovakia
}

\begin{abstract}
The set of 25 inorganic porous building materials was put through the cumulative water inflow and drying tests in laboratory conditions. Besides these experiments the parameters, including pore structure parameters of the tested materials have been determined. The characteristics of the capillary suction induced water absorption - the water absorption coefficient, and drying - the initial constant drying rate, were compared with their analytical expressions. The results of this analysis show the measure of particular material parameters and boundary conditions significance for the coincidence of experimental and calculation results. At the drying an important correlation between experimentally obtained initial water content and drying rate was observed.
\end{abstract}

\section{Introduction}

The moisture transport in porous building materials for one dimensional problem is modelled by the diffusion equation in the form:

$$
\frac{\partial w}{\partial t}=\frac{\partial}{\partial x}\left[D(w) \cdot \frac{\partial w}{\partial x}\right]
$$

Where $D(w)$ is the moisture diffusivity $\left(\mathrm{m}^{2} / \mathrm{s}\right)$ which is a function of the moisture content $w\left(\mathrm{~kg} / \mathrm{m}^{3}\right), t$ is the time (s) and $x$ is the space coordinate (m).

There are two basic types of boundary conditions at solution of Equation (1): 1. the contact of dry material surface with liquid water, causing the process of instantaneous water suction into the material, 2. the contact of a wet material surface with humid air environment, causing the process of material drying. The analytical solutions of time dependent cumulative moisture content at these two moisture transport modes were compared with the results of suction and drying experiments in laboratory conditions. The experiments were realised in the $23^{\circ} \mathrm{C}$ and $50 \%$ relative humidity environment using the prismatic specimens for suction and the thin plate specimens for drying. The experiments were complemented with the mercury intrusion porosimetry tests of all the analysed materials.

\section{Water suction}

At the solution of Equation (1) for capillary suction inducted water absorption the initial conditions for semiinfinite solid are:

\footnotetext{
Corresponding author: usarmat@savba.sk
}

$$
w(t=0, x \geq 0)=w_{\text {dry }}
$$

The contact of solid material surface with liquid water is expressed by the boundary conditions of the first kind (Dirichlet type):

$$
w(t>0, x=0)=w_{\text {sat }}
$$

The cumulative water inflow during water absorption has the form:

$$
w(t)=A \cdot t^{0.5}
$$

and this process ends when the material reaches a capillary moisture content. The capillary moisture content is the value obtained in a short-term capillary rise experiment when the air initially present in the pores is partly entrapped in the largest pores preventing absorption until the full water saturation state [1].

The slope of function (3) is the water absorption coefficient $A\left(\mathrm{~kg} / \mathrm{m}^{2} \cdot \mathrm{s}^{0.5}\right)$. It is defined by the relation resulting from the information on the moisture content profiles during the absorption experiment [2]:

$$
A=\rho_{\mathrm{w}} \cdot w_{\text {cap }} \int_{0}^{1} \lambda d s
$$

where $\rho_{\mathrm{w}}$ is the bulk density of water $\left(\mathrm{kg} / \mathrm{m}^{3}\right), w_{\text {cap }}$ is the capillary moisture content $\left(\mathrm{m}^{3} / \mathrm{m}^{3}\right), \lambda$ is the Boltzmann coordinate $x / t^{-0.5}\left(\mathrm{~m} / \mathrm{s}^{0.5}\right)$ and $s$ is the degree of saturation (-). In case when the information on the moisture profiles is not available the absorption coefficient can be approximated by the sharp penetration profile model where the real moisture profile is 
simplified to the sharp moisture profile. The position of water penetration front is expressed as the equivalent capillary meniscus position. The water absorption coefficient is then characterized by the capillary moisture content and the effective pore radius represented by the hydraulic radius $r_{\mathrm{H}}(\mathrm{m}), \sigma$ is the surface tension of water $(\mathrm{N} / \mathrm{m})$ and $\eta$ is the dynamic viscosity of water (Pa.s) [3]:

$$
A=\rho_{\mathrm{w}} \cdot w_{\text {cap }} \cdot \sqrt{\frac{r_{\mathrm{H}} \cdot \sigma_{\mathrm{lg}}}{2 \cdot \eta}}
$$

In Fig. 1. the comparison of the measured water absorption coefficients with the results of Equation (5) calculated from the measured input data shows a discrepancy between calculated and measured values. The sharp penetration profile model is too simple to be valid for all the analysed materials.

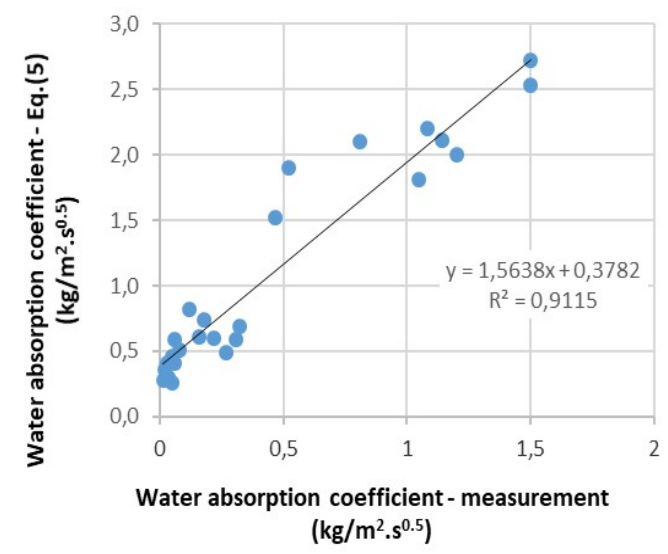

Fig. 1. Relationship between measured and calculated water absorption coefficient - (Eq. 5).

A more specific approach is to approximate the water absorption coefficient by the equivalent capillary model under the assumption, that the expression of the absorption coefficient contains a parameter - correction factor [4]. This factor is expressed by a power function of the pore volume effective in the transport, given by the difference between capillary and critical moisture content $w_{\text {crit }}\left(\mathrm{m}^{3} / \mathrm{m}^{3}\right)$ :

$$
A=\rho_{w} \cdot w_{c a p} \cdot \sqrt{\frac{0.76 \cdot\left(w_{c a p}-w_{c r i t}\right)^{3}}{w_{c a p}} \cdot \frac{r_{H} \cdot \sigma_{\mathrm{lg}}}{2 \cdot \eta}}
$$

The critical moisture content is defined as the lowest moisture content necessary to initiate moisture transport in the liquid phase [5].

In Figure 2 the comparison of the measured data with the results of Equation (6) indicates that the equation is an acceptable formula for calculating the water absorption coefficient, if we know the values of capillary and critical moisture contents and hydraulic radii. The critical moisture contents were determined from drying tests and the hydraulic radii were determined from mercury intrusion porosimetry tests.

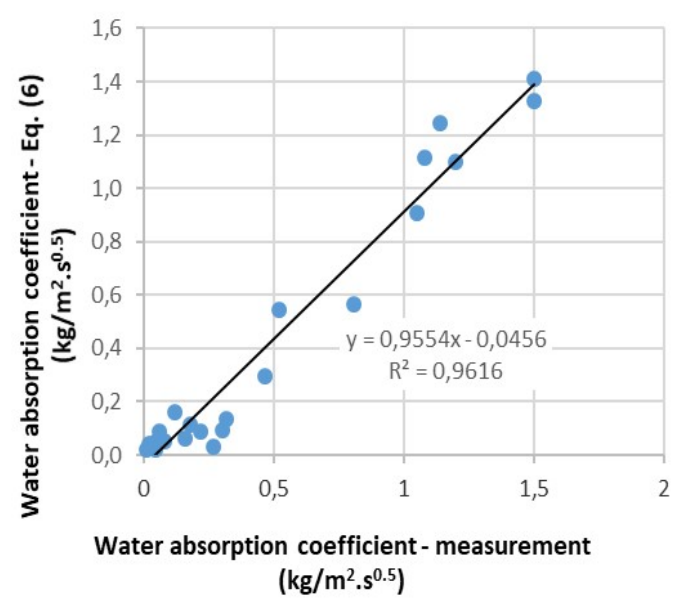

Fig. 2. Relationship between measured and calculated water absorption coefficient - Eq. (6).

The capillary moisture content is related with the pore structure parameters determined by mercury intrusion porosimetry and it can be effectively substituted by the difference between porosity and the volume critical for mercury intrusion process. The comparison of the calculations using this substitution with the measurements gives the coefficient of determination equal to 0.967 . The use of the average critical volume 0.12 gives the coefficient of determination equal to 0.963 .

\section{Drying}

The drying process is significantly slower process in comparison with the water absorption. During the drying in general two basic periods can be distinguished [6]. The first drying period starts at the capillary moisture content and it lasts until reaching the critical (residual) liquid moisture content. In the second drying phase the time course is determined by decreasing difference between material and surrounding water vapour pressures and the drying rate is decreasing, starting at the critical moisture content. In this paper the first drying period is analysed.

This first period is determined mainly by the boundary conditions, which are given by the water evaporation from a wet material surface with constant water vapour pressure and also by the material properties. The drying course is linear and the drying rate is constant during this period.

During drying the initial conditions for infinite plate of thickness $d(\mathrm{~m})$ are:

$$
w(t=0,0<x<d)=w_{\text {cap }}
$$

where $w_{\text {cap }}$ is the capillary moisture content $(\mathrm{kg} / \mathrm{m} 3)$.

During the first drying period the constant water flow rate density $q\left(\mathrm{~kg} / \mathrm{m}^{2} . \mathrm{s}\right)$ is used, as a symetrical boundary condition of the second kind (Neumann type):

$$
D(w) \cdot \frac{\partial w}{\partial x}=q \quad(t>0, x=0, x=d)
$$


Under defined boundary conditions the analytical solution of Equation (1) for average moisture content time course is [7]:

$$
\bar{w}(t)=w_{\text {cap }}-\frac{q \cdot t}{(d / 2)}
$$

The moisture diffusivity is so high that there is no moisture profile during this period. The drying rate is proportional to the difference between capillary and critical moisture contents and inversely proportional to the duration of the first period. It can be expressed as the reduction (relative evaporation $G$ ) of the wet surface evaporation potential $q_{\mathrm{p}}$ :

$$
q=\frac{\left(w_{\text {cap }}-w_{\text {crit }}\right) \cdot(d / 2)}{t_{\mathrm{d}}}=G \cdot q_{\mathrm{p}}
$$

where $w_{\text {crit }}$ is the critical moisture content or irreducible liqid water saturation $\left(\mathrm{kg} / \mathrm{m}^{3}\right), t_{\mathrm{d}}(\mathrm{s})$ is the duration of the first drying period.

The relative evaporation is the ratio of actual to potential evaporation [8]. The evaporation potential is calculated as:

$$
q_{\mathrm{p}}=\beta \cdot\left(p_{\mathrm{sat}}-p\right)
$$

$\beta$ is the surface water vapour diffusion coefficient $\left(\mathrm{kg} / \mathrm{m}^{2} . \mathrm{s} . \mathrm{Pa}\right), p_{\text {sat }}$ is the saturation water vapour pressure $(\mathrm{Pa}), p$ is the ambient water vapour pressure.

The drying rate during the constant period can be expressed also by the boundary conditions of the third kind (Robin boundary conditions) which are expressed by the constant coefficient $\beta$ and the surface vapour pressure $p_{\text {surf }}$ approximately constant during this period:

$$
q=\beta \cdot\left(p_{\text {surf }}-p\right)
$$

The results of drying rate density calculated by Equation (9) using the parameters determined in drying experiments, are compared with the measured data in Fig. 3. Their coincidence is acceptable.

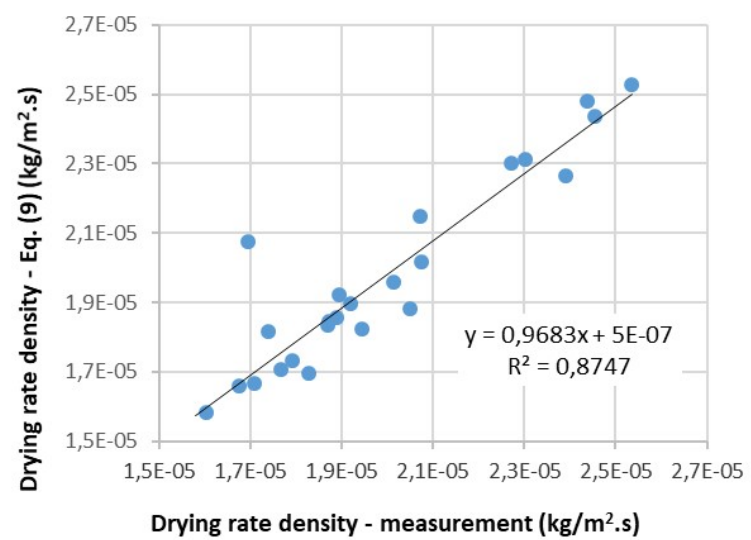

Fig. 3. Relationship between measured and calculated drying rate density - Eq. (9).
In comparison with the relationship for calculation of the water absorption coefficient, where all input parameters are the parameters of pore structure, the determination of constant drying rate is dependent also on the duration of constant drying period. The possibility to determine this parameter independently on the experiment as the parameter of given material specimen properties would be useful.

A strong correlation was found between the durations of constant drying period and the initial water contents of drying plates, obtained from the experiments, depicted in Figure 4. The correlation covers the capillary moisture contents in interval $0.14-0.87 \mathrm{~m}^{3} / \mathrm{m}^{3}$ and the plates thicknesses in interval $0.006-0.02 \mathrm{~m}$.

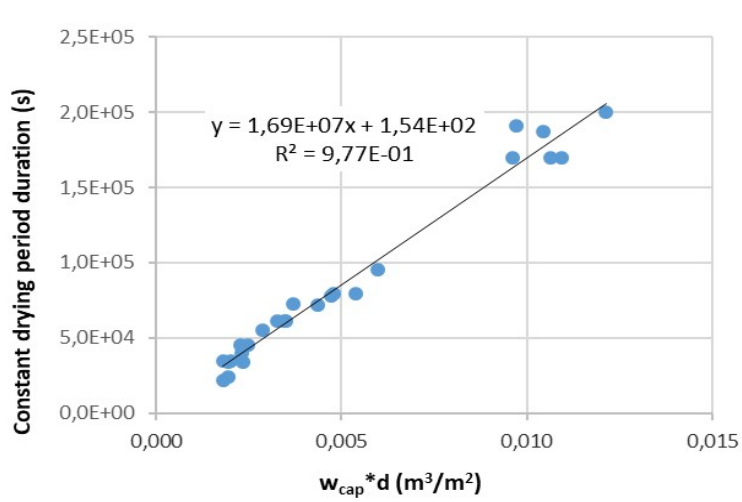

Fig. 4. Relationship between initial water and duration of constant drying period.

Insertion of the regression relationship from Fig. 4.:

$$
t_{\mathrm{d}}=1.69 \cdot 10^{7} .\left(w_{\text {cap }} . d\right)+1.54 \cdot 10^{2}
$$

in Eq. (9) gives the results compared with the measurements in Fig. 5. Their coincidence with the results of mesurements is weaker than in case given by Eq. (9) using the measured duration data. In spite of the significance of the correlation (12) the relationship (9) is apparently very sensitive to the estimation of constant drying period duration values.

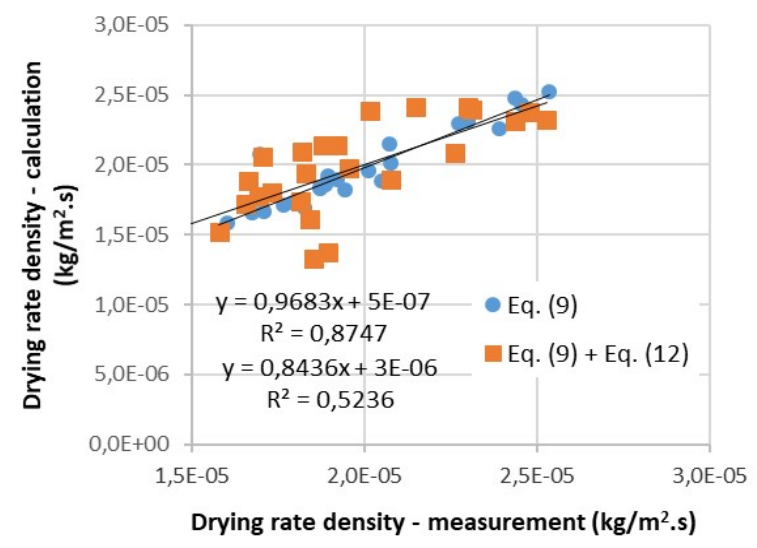

Fig. 5. Relationship between measured drying rate densities and the drying rate densities calculated by Eq. (9) and Eqs. (9) and (12). 


\section{Conclusions}

Parameters of the water absorption - water absorption coefficient, and the drying - constant drying rate density were determined by the laboratory experiments of suction and drying the inorganic porous building materials. Besides, the pore structure parameters by mercury intrusion porosimetry method were determined.

Time courses of the cumulative water inflow and the drying time courses were expressed by analytical solutions of moisture diffusion equation. As the input data the parameters obtained from the experiments: capillary moisture content, critical moisture content, hydraulic radius, duration of constant drying rate period were used in the calculations of the material characteristics - water absorption coefficient and drying rate density.

The analysed water absorption coefficient and drying rate density are the functions of pore structure parameters: capillary and critical moisture contents obtained from moisture transport experiments.

The equivalents of these parameters can be determined from the results of mercury intrusion porosimetry tests: the porosity and the critical volume of the mercury intrusion process.

Specific parameters for calculations are the hydraulic radius, the parameter of absorption, which can be determined from the porosimetry and the duration of constant drying period, which is proportional to the initial water content of drying material.

In general, the equivalence of real moisture transport processes with their calculations is dependent on the reliability of input parameters determination as well as on the reliability of time dependent moisture content measurements during the water absorption and drying experiments.

The authors greatly acknowledge the financial support by the Slovak Research and Development Agency under contract No. APVV-15-0631.

\section{References}

1. F. Descamps, F. PhD-dissertation K.U. Leuven, Leuven (1997)

2. R.J. Gummerson, C. Hall, W.D. Hoff, Building and Environment, 15 (1980)

3. Ch. Hall, W. Hoff. Taylor \& Francis, New York, (2002)

4. P. Matiašovský, O. Koronthályová, Research in Building Physics and Building Engineering. Concordia University, Montreal (2006)

5. M.K. Kumaran, Final Report. Task 3: Material Properties. IEA Annex 24, K.U. Leuven (1996).

6. A.S. Mujumdar, S. Devahastin. Exergex Corporation (2000).

7. H.S. Carslaw, J. C. Jaeger. Oxford at the Clarendon Press (1959).

8. R.J. Granger, D.M. Gray, Journal of Hydrologv 111, (1989) 